\title{
Trabalho, atividades de lazer e apoio familiar: fatores para proteção da qualidade de vida de idosos
}

\section{Work, leisure activities and family support: factors to protect quality of life of elderly}

\author{
Maysa de Oliveira Silva ${ }^{1}$, Álvaro da Silva Santos ${ }^{2}$, Lucila Costa Zini \\ Angelotti $^{3}$, Valéria Sousa de Andrade ${ }^{4}$, Grasielle Silveira Tavares ${ }^{5}$
}

http://dx.doi.org/10.11606/issn.2238-6149.v28i2p163-172

Silva MO, Santos AS, Angelotti LCZ, Andrade VS, Tavares GS. Trabalho, atividades de lazer e apoio familiar: fatores para proteção da qualidade de vida de idosos residentes no município de SacramentoMG. Rev Ter Ocup Univ São Paulo. 2017 maio-ago.;28(2):163-72.

RESUMO: Este estudo objetivou verificar a influência do trabalho, apoio familiar e das atividades de lazer na qualidade de vida (QV) de idosos. A amostra foi composta por 169 idosos residentes no município de Sacramento-MG com idade $\geq$ a 60 anos. Foram utilizados os instrumentos WHOQOL-Brefe WHOQOL-Old para avaliação da QV. Informações acerca do trabalho, apoio familiar e lazer foram coletadas por meio de questionários semiestruturados. Os dados foram analisados pelos testes t de Student e Mann Whitney, considerando-se $\mathrm{p}<0,05$. As atividades de lazer correlacionaram-se com domínios "físico", "psicológico" e "global" do WHOQOL-Bref e, com "funcionamento dos sentidos", "autonomia", "participação social" e com escore global do WHOQOL-Old. Os aposentados que ainda trabalham tiveram escore do domínio "físico" e na faceta "morte e morrer" significativamente mais altos em relação aos idosos que não trabalham mais. $\mathrm{O}$ apoio social configurou como importante estratégia para o enfrentamento das adversidades cotidianas, sendo que o convívio familiar mostrou ser um fator com efeito significativo sobre os escores médios de QV em quase todos os domínios dos instrumentos. O bom convívio familiar, o trabalho remunerado e a participação em atividades de lazer podem ser considerados fatores de proteção para QV dos idosos entrevistados.

DESCRITORES: Idoso; Família; Qualidade de vida; Atividades de lazer; Trabalho.
Silva MO, Santos AS, Angelotti LCZ, Andrade VS, Tavares GS. Work, leisure activities and family support: factors to protect quality of life of elderly. Rev Ter Ocup Univ São Paulo. 2017 May-Aug.;28(2):163-72.

ABSTRACT: This study aimed to investigate the influence of work, family support and leisure activities on the quality of life (QOL) of elderly. The sample consisted of 169 elderly residents in Sacramento, MG aged 60 years old or more. In order to evaluate QOL it was used the WHOQOLBref e WHOQOL-Old. Information regarding work, family support, leisure activities and work was collected through semi-structured questionnaires. Data were analyzed by using the Student t test and Mann-Whitney, considering $\mathrm{p}<0.05$. Leisure activities were related to the "physical", "psychological" and "global" domains of WHOQOLBref and to the "sensory abilities", "autonomy", "social participation" facets and overall score of WHOQOL-Old. Retirees who still worked had significantly higher scores on the domain "physical" and on the facet "death and dying" compared to ones who did no work anymore. Social support was an important strategy to coping with daily problems. Good family life, paid work and participation in leisure activities can be considered protective factors for the quality of life of seniors.

KEYWORDS: Aged, Family, Quality of life, Leisure activities, Work.

1. Terapeuta Ocupacional, Recanto Geriátrico de Passos, e Hospital psiquiátrico Otto Krakauer. E-mail: ysamix13@hotmail.com

2. Enfermeiro, Doutor em Ciências Sociais pela Pontifícia Universidade Católica de São Paulo, docente da Universidade Federal do Triângulo Mineiro, UFTM. E-mail: alvaroenf@hotmail.com

3. Estatística, Mestre em Inovação Tecnológica, docente da Universidade Federal do Triângulo Mineiro, UFTM. E-mail: lucila.zini@gmail.com

4. Terapeuta Ocupacional, Mestre na Ciência de Reabilitação, docente da Universidade Federal do Triângulo Mineiro, UFTM. E-mail: valeria-to@hotmail.com

5. Terapeuta Ocupacional, Doutora em Saúde Publica pela USP, docente do curso de Terapia Ocupacional da Universidade de Brasília UNB. E-mail: grasiellet@yahoo.com.br

O material é parte do trabalho de conclusão de curso exigido como requisito para graduação em Terapia Ocupacional. Esta contribuição é original e inédita. Seu texto não está sendo avaliado para publicação por outra revista.

Fonte de financiamento: Fundação de Amparo à Pesquisa do Estado de Minas Gerais - FAPEMIG.

Endereço para correspondência: Valéria Sousa de Andrade. Universidade Federal do Triângulo Mineiro/UFTM. Av. Frei Paulino. Bairro Nossa Senhora da Abadia, Uberaba, MG, Brasil. CEP: 38025-180. 


\section{INTRODUÇÃO}

$\mathrm{O}$ envelhecimento populacional vem se tornando um fenômeno em evidência nas últimas décadas devido ao aumento na expectativa de vida, ao incremento das tecnologias e pesquisas e à diminuição da taxa de natalidade ${ }^{1}$. Essa transformação sociodemográfica implica na necessidade de uma nova configuração dos sistemas de saúde para atender ao aumento da demanda de atendimentos e às necessidades da população idosa $a^{2,3,4}$.

A mudança da configuração etária contribuiu para a transição epidemiológica de doenças agudas transmissíveis para doenças crônicas não transmissíveis ${ }^{5}$, que interferem na funcionalidade, participação e autonomia, demandando maior apoio familiar 6 . Embora o idoso frequentemente apresente condições crônicas de saúde, mantem sua funcionalidade e qualidade de vida $(\mathrm{QV})$ desde que haja prevenção e controle de agravos e de incapacidades?

Esses aspectos relacionam-se ao processo do envelhecimento ativo que implica envelhecer com participação e engajamento na vida e na sociedade, otimizando as oportunidades do idoso em se manter saudável $^{8}$, desejo este que se torna imprescindível para a obtenção de melhor QV.

A QV é descrita como a percepção do indivíduo sobre sua posição na vida/cultura/relação conforme suas metas, expectativas, padrões e sistema de valores ${ }^{7}$. Destarte, para sua avaliação, torna-se importante considerar a saúde física bem como estado psicoemocional, relações sociais, nível de autonomia, meio ambiente e questões referentes às crenças e espiritualidade ${ }^{1}$.

A manutenção das relações sociais configura-se como importante estratégia para o auxílio no enfrentamento das adversidades cotidianas e do sentimento de solidão. Assim, a família se torna a principal rede de suporte para o idoso, sendo o bom convívio com a mesma um fator importante para seu bem-estar e $\mathrm{QV}^{9}$.

Dentro dessa perspectiva, a literatura constata o efeito protetor de fatores como manutenção de relações sociais, para a funcionalidade e sua contribuição para o envelhecimento ativo ${ }^{10}$. Considera-se que também o trabalho remunerado e o lazer são relevantes para o bemestar no envelhecimento. Contrariamente, o processo de aposentadoria pode implicar muitas vezes em um complicador da vivência do processo de envelhecimento, uma vez que aposentar-se significa o afastamento do trabalho, quebra de um cotidiano, rompimento de vínculos e associa-se com sentimentos de perdas e inutilidade, pois o trabalho tem papel de regulador da organização humana, capaz de promover desenvolvimento pessoal e reconhecimento social ${ }^{11}$.

Quanto ao lazer, entendido como uma forma de ocupação com participação voluntária e desinteressada, torna-se capaz de proporcionar prazeres e descanso bem como condições de recuperação psicossomática, amenizando assim impactos do envelhecimento, refletindo sobre a QV e sentimentos de auto-realização ${ }^{12}$.

Com base no exposto, objetivou-se verificar a influência do trabalho remunerado após a aposentadoria, o engajamento de idosos em atividades de lazer e o apoio familiar na QV de idosos. A investigação destas variáveis independentes pautou-se no interesse em verificar como se ocupações, especificamente, o lazer, a participação social e o trabalho, podem contribuir com o bem-estar e qualidade de vida de uma população especifica de idosos.

\section{MÉTODOS}

Trata-se de um estudo descritivo, exploratório, de corte transversal realizado a partir de um projeto de pesquisa financiado pela Fundação de Amparo à Pesquisa do Estado de Minas Gerais/FAPEMIG denominado "Perfil de Saúde da População idosa dos municípios da gerência regional de saúde de Uberaba/MG". Os dados considerados se referem aos idosos residentes no município de Sacramento-MG, cidade com aproximadamente 26.000 habitantes, dentre os quais aproximadamente 3.000 eram indivíduos a partir de 60 anos $^{13}$.

Foram elegíveis a participar do estudo indivíduos com idade igual ou superior a 60 anos de idade, cadastrados nas Unidades Básicas de Saúde (UBS) da região urbana e zona rural, capazes de deambular (com ou sem auxílio de equipamento de autoajuda) e que apresentaram condições cognitivas consideradas normais no Mini Exame do Estado Mental (MEEM) em conformidade com o nível de escolaridade. Optou-se por adotar o MEEM visto tratar-se de um instrumento validado e padronizado frequentemente utilizado para rastreio e avaliação cognitiva, o que favoreceu a seleção de idosos aptos a compreender e responder os instrumentos utilizados na pesquisa ${ }^{14}$. Excluiu-se indivíduos com déficit grave de audição e/ou visão que dificultasse a comunicação para coleta dos dados bem como aqueles que se caracterizavam como cadeirantes ou acamados, posto que o projeto inicial solicitou a execução de registros de medidas antropométricas que poderiam ser prejudicadas pelas condições mencionadas. A partir de amostragem por conveniência foram selecionados os participantes do estudo, tendo correspondido aos idosos residentes no município, que frequentaram as UBS no período da coleta de dados, 
ocorrida no período de maio de 2012 a abril de 2013. As entrevistas para tal se deram de forma individual e foram realizadas por profissionais previamente treinados.

Para avaliar o perfil da população foram utilizados questionários semiestruturados sobre situação sociodemográfica, apoio familiar, trabalho remunerado após aposentadoria e atividades de lazer. Visando possibilitar a análise dos dados, as respostas às questões que investigavam as atividades de lazer foram categorizadas em até quatro atividades e cinco ou mais. Referente ao "apoio familiar" investigou-se a situação conjugal dos idosos, presença de filhos, e moradia com filhos ou recebimento de alguma cooperação financeira dos familiares. Sobre o "convívio familiar", as respostas foram categorizadas em duas opções (ótimo ou bom e ruim ou regular). Quanto ao "trabalho após a aposentadoria" as questões foram combinadas originando quatro grupos: aposentados que trabalham; aposentados que não trabalham; não aposentados que ainda trabalham e não aposentados que não trabalham. Destes foram considerados para a comparação de médias os grupos que continham idosos aposentados.

Para avaliar QV foram utilizados o WHOQOL-Bref e o WHOQOL-Old, ambos validados para a população brasileira $^{18}$. O WHOQOL-Bref, forma abreviada do WHOQOL 100, é composto por 26 questões distribuídas em quatro domínios (físico, psicológico, relações sociais e meio ambiente) ${ }^{15,16}$. O WHOQOL-Old possui 24 questões que se distribuem em seis facetas (funcionamento dos sentidos; autonomia; atividades passadas, presentes e futuras; participação social; morte e morrer e, intimidade ${ }^{17}$. As respostas são dadas através de uma escala do tipo Likert graduada em cinco níveis para cada pergunta. Para o agrupamento das questões de acordo com os domínios do WHOQOL-Bref, as facetas do WHOQOL-Old e a computação dos respectivos escores, foram utilizadas as sintaxes para o $\mathrm{SPSS}^{20}$, nas quais os escores foram transformados em uma escala de 0 a 100 , sendo que quanto mais elevado os escores melhor a $\mathrm{QV}$, não sendo estimado ponto de corte dos instrumentos.

Os dados foram processados em uma planilha no Excel, sendo digitados em dupla entrada. Para análise dos dados o banco foi importado para o software SPSS (versão 20). As variáveis sociodemográficas, apoio familiar, trabalho remunerado e atividades de lazer foram analisadas de forma descritiva em termos de frequência absoluta e relativa. Para as variáveis "idade, anos de estudo, escores da QV" foram utilizadas medidas de tendência central (média) e variabilidade (desvio padrão). O teste Kolmogorov-Smirnov foi utilizado para verificar a normalidade dos escores da QV nas diferentes dimensões. A comparação das médias dos escores de qualidade de vida em relação às variáveis "trabalho remunerado após a aposentadoria, atividades de lazer, apoio familiar" foi realizada através dos testes t de Student e Mann Whitney. Foi considerado erro amostral de 0,05 e intervalo de confiança de $95 \%$.

O projeto de pesquisa foi aprovado pelo Comitê de Ética em Pesquisa (CEP/UFTM) sob protocolo de número 1640/2010. Todos os participantes assinaram o termo de consentimento livre e esclarecido (TCLE).

\section{RESULTADOS}

Participaram deste estudo 169 idosos, $116(68,6 \%)$ do sexo feminino e $53(31,4 \%)$ masculino. A idade variou de 60 a 90 anos $(\mu: 69,47)$, predominando participantes com 60-69 anos (53,3\%). Os idosos entrevistados demonstraram haver estudado entre 0 e 23 anos, sendo que $62,7 \%$ deles tinham de 1 a 4 anos de estudo e $6,5 \%$ nove anos ou mais. A maioria $(64,3 \%)$ relatou ter renda familiar maior que um e menor ou igual a três salários mínimos (SM). A Tabela 1 ilustra os dados sociodemográficos.

Tabela 1 - Distribuição das características sociodemográficas $(\mathrm{n}=169)$

\begin{tabular}{lcc}
\hline Característica & $\mathbf{N}$ & $\mathbf{\%}$ \\
\hline Sexo & 116 & 68,6 \\
Feminino & 53 & 31,4 \\
Masculino & & \\
Faixa etária & 90 & 53,3 \\
$60-69$ & 65 & 38,5 \\
$70-79$ & 14 & 8,2 \\
$80+$ & & \\
Anos de estudo & 37 & 21,9 \\
0 & 106 & 62,7 \\
1 a 4 & 15 & 8,9 \\
5 a 8 & 11 & 6,5 \\
$9+$ & & \\
Renda familiar* & 16 & 9,5 \\
$<1$ SM & 14 & 8,3 \\
1 SM & 108 & 64,3 \\
$>1 \leq 3$ SM & 22 & 13,1 \\
$>3 \leq 7$ SM & 8 & 4,8 \\
$>7$ SM & & \\
\hline
\end{tabular}

*Um participante não respondeu à pergunta, totalizando 168 sujeitos; SM: salário(s) mínimo(s); $<<0,05$. 
Os resultados relativos ao trabalho remunerado e à aposentadoria apontam que $52(31 \%)$ participantes eram aposentados e possuíam um trabalho ou ocupação remunerada; $9(5,3 \%)$ trabalhavam e ainda não estavam aposentados; $22(13,1 \%)$ não estavam aposentados e não trabalhavam; e 85 (50,6\%) eram aposentados, já não exerciam nenhuma atividade remunerada. Os idosos que ainda exerciam alguma atividade remunerada após a aposentadoria apresentaram escores médios significativamente mais altos nos domínios "físico" do WHOQOL-Brefe " morte e morrer" do WHOQOL-Old em relação aos idosos que não exerciam tal ocupação. A Tabela 2 exibe os fatores investigados como sendo possíveis influenciadores de proteção para QV que compreendem o trabalho remunerado após aposentadoria, apoio familiar e atividades de lazer.

Tabela 2 - Distribuição das características investigadas como fatores de proteção para a QV (n=169)

\begin{tabular}{|c|c|c|}
\hline Característica & $\mathbf{N}$ & $\%$ \\
\hline \multicolumn{3}{|l|}{ Trabalho/aposentadoria* } \\
\hline A-T & 52 & 31,0 \\
\hline A-NT & 85 & 50,6 \\
\hline NA-T & 9 & 5,3 \\
\hline NA-NT & 22 & 13,1 \\
\hline \multicolumn{3}{|l|}{ Situação conjugal* } \\
\hline Vive com o(a) parceiro(a) & 110 & 65,5 \\
\hline Vive sem o(a) parceiro(a) & 58 & 34,5 \\
\hline \multicolumn{3}{|l|}{ Filhos* } \\
\hline Sim & 158 & 94,0 \\
\hline Não & 10 & 6,0 \\
\hline \multicolumn{3}{|l|}{ Mora com Filhos* } \\
\hline Sim & 64 & 40,5 \\
\hline Não & 94 & 59,5 \\
\hline \multicolumn{3}{|l|}{ Ajuda financeira } \\
\hline Sim & 71 & 42,0 \\
\hline Não & 98 & 59,4 \\
\hline \multicolumn{3}{|l|}{ Convívio familiar } \\
\hline Ótimo/Bom & 150 & 88,8 \\
\hline Regular/ruim & 19 & 11,2 \\
\hline \multicolumn{3}{|l|}{ Atividades de lazer } \\
\hline 0 a 4 & 114 & 67,5 \\
\hline $5+$ & 55 & 32,5 \\
\hline
\end{tabular}

Com base na Tabela 2, em relação ao estado conjugal verificou-se que $110(65,5 \%)$ viviam com um parceiro e que $158(94,0 \%)$ tinham filhos, destes, 64 (40,5\%) moravam com os filhos, $71(42,0 \%)$ recebiam ajuda financeira dos familiares, $150(88,8 \%)$ tinham bom ou ótimo contato familiar, e $19(11,2 \%)$ declararam convívio familiar regular ou ruim. Em relação às atividades de lazer, 114 (67,5\%) explicitaram realizar até quatro atividades de lazer em seu cotidiano e 55 (32,5\%) cinco ou mais atividades de lazer.

A comparação de médias dos escores da $\mathrm{QV}$ do instrumento WHOQOL-Bref em relação ao engajamento em trabalho remunerado, apoio familiar e atividades de lazer encontra-se sintetizada na Tabela 3.

Tabela 3 - Comparação das médias dos domínios e média global de QV do instrumento WHOQOL-Bref em função das variáveis "atividades de lazer", "apoio familiar" e "trabalho remunerado após a aposentadoria” (n=169)

\begin{tabular}{|c|c|c|c|}
\hline Domínios & \multicolumn{2}{|c|}{ Característica } & p-valor \\
\hline & \multicolumn{2}{|c|}{ Atividades de lazer } & \\
\hline & 0 a 4 & $5+$ & \\
\hline Físico & $61,69 \pm 16,85$ & $67,20 \pm 14,75$ & $0,040 *$ \\
\hline Psicológico & $65,49 \pm 15,21$ & $73,25 \pm 11,12$ & $0,002 * *$ \\
\hline Relações sociais & $77,79 \pm 15,56$ & $80,85 \pm 13,17$ & 0,319 \\
\hline Meio ambiente & $60,79 \pm 15,38$ & $65,56 \pm 13,74$ & 0,063 \\
\hline \multirow[t]{3}{*}{ Global } & $64,12 \pm 20,78$ & $73,44 \pm 14,58$ & $0,005 * *$ \\
\hline & \multicolumn{2}{|c|}{$\begin{array}{c}\text { Ter filhos } \\
\end{array}$} & \\
\hline & Sim & Não & \\
\hline Físico & $63,26 \pm 16,38$ & $69,10 \pm 15,28$ & 0,274 \\
\hline Psicológico & $67,77 \pm 14,26$ & $72,90 \pm 17,52$ & 0,409 \\
\hline Relações sociais & $78,83 \pm 15,00$ & $80,20 \pm 11,87$ & 0,967 \\
\hline Meio ambiente & $62,20 \pm 18,27$ & $65,80 \pm 18,27$ & 0,393 \\
\hline \multirow[t]{3}{*}{ Global } & $66,88 \pm 19,51$ & $71,20 \pm 19,68$ & 0,400 \\
\hline & \multicolumn{2}{|c|}{ Morar com filhos } & \\
\hline & Sim & Não & \\
\hline Físico & $64,11 \pm 16,61$ & $62,47 \pm 16,32$ & 0,539 \\
\hline Psicológico & $67,28 \pm 13,96$ & $68,00 \pm 14,49$ & 0,632 \\
\hline Relações sociais & $78,16 \pm 16,48$ & $79,06 \pm 14,07$ & 0,933 \\
\hline Meio ambiente & $60,53 \pm 14,66$ & $63,20 \pm 14,88$ & 0,381 \\
\hline \multirow[t]{3}{*}{ Global } & $65,83 \pm 18,99$ & $67,62 \pm 19,84$ & 0,573 \\
\hline & \multicolumn{2}{|c|}{ Cooperação financeira familiar } & \\
\hline & Sim & Não & \\
\hline Físico & $61,14 \pm 16,79$ & $65,18 \pm 15,91$ & 0,113 \\
\hline Psicológico & $67,87 \pm 14,59$ & $78,07 \pm 15,20$ & 0,923 \\
\hline
\end{tabular}


Silva MO, et al. Trabalho, atividades de lazer e apoio familiar. Rev Ter Ocup Univ São Paulo. 2017 maio/ago.;28(2):163-72.

Tabela 3 - Comparação das médias dos domínios e média global de QV do instrumento WHOQOL-Bref em função das variáveis "atividades de lazer", "apoio familiar" e "trabalho remunerado após a aposentadoria" ( $\mathrm{n}=169)$

\begin{tabular}{|c|c|c|c|}
\hline \multirow{2}{*}{$\begin{array}{l}\text { Domínios } \\
\text { Relações sociais }\end{array}$} & \multicolumn{2}{|c|}{ Característica } & \multirow{2}{*}{$\begin{array}{c}\text { p-valor } \\
0,551\end{array}$} \\
\hline & $79,77 \pm 14,41$ & $68,12 \pm 14,42$ & \\
\hline Meio ambiente & $62,30 \pm 13,25$ & $62,38 \pm 16,21$ & 0,961 \\
\hline \multirow[t]{3}{*}{ Global } & $68,14 \pm 19,19$ & $66,44 \pm 19,73$ & 0,434 \\
\hline & \multicolumn{2}{|c|}{ Convívio familiar } & \\
\hline & Ótimo/bom & Regular/ruim & \\
\hline Físico & $64,69 \pm 16,62$ & $54 \pm 17,32$ & 0,007 \\
\hline Psicológico & $69,50 \pm 13,80$ & $56,32 \pm 14,43$ & 0,000 \\
\hline Relações sociais & $80,81 \pm 12,76$ & $62,84 \pm 20,20$ & 0,000 \\
\hline Meio ambiente & $64,16 \pm 14,07$ & $48,00 \pm 14,63$ & 0,000 \\
\hline \multirow[t]{3}{*}{ Global } & $68,51 \pm 19,38$ & $56,47 \pm 16,86$ & 0,003 \\
\hline & \multicolumn{2}{|c|}{ Situação conjugal } & \\
\hline & Com parceiro & Sem parceiro & \\
\hline Físico & $64,73 \pm 16,62$ & $60,86 \pm 15,68$ & 0,146 \\
\hline Psicológico & $67,77 \pm 13,85$ & $68,29 \pm 15,67$ & 0,67 \\
\hline Relações sociais & $79,28 \pm 15,19$ & $77,78 \pm 14,39$ & 0,412 \\
\hline Meio ambiente & $62,83 \pm 15,18$ & $61,53 \pm 14,84$ & 0,855 \\
\hline \multirow[t]{3}{*}{ Global } & $66,71 \pm 18,92$ & $67,64 \pm 20,53$ & 0,502 \\
\hline & \multicolumn{2}{|c|}{ Ocupação remunerada } & \\
\hline & A-T & A-NT & \\
\hline Físico & $67,73 \pm 13,90$ & $61,32 \pm 15,90$ & 0,018 \\
\hline Psicológico & $71,52 \pm 10,82$ & $67,68 \pm 15,14$ & 0,102 \\
\hline Relações sociais & $82,10 \pm 12,39$ & $77,48 \pm 14,14$ & 0,073 \\
\hline Meio ambiente & $63,40 \pm 14,87$ & $62,27 \pm 14,96$ & 0,773 \\
\hline Global & $70,88 \pm 15,93$ & $66,73 \pm 18,99$ & 0,162 \\
\hline
\end{tabular}

A-T: aposentados que trabalham; A-NT: aposentados que não trabalham; *Comparação de médias conforme o teste $\mathrm{t}$ de Student; **Comparação de tendências centrais conforme o Mann Whitney.

Verificou-se, como apresentado na Tabela 3 que os idosos que realizavam cinco ou mais atividades de lazer cotidianamente tiveram melhores escores com diferenças significativas nos domínios "físico, psicológico e global" do WHOQOL-Bref, A Tabela 4 exibe informações sobre a comparação das médias das facetas de QV do instrumento WHOQOL-Old em função das variáveis "atividades de lazer", "apoio familiar" e "trabalho remunerado após a aposentadoria" e revela que aqueles com cinco ou mais atividades de lazer tiveram melhores escores nas facetas "funcionamento dos sentidos, autonomia, participação social" e no "escore global" do WHOQOL-Old quando comparados àqueles que realizam quatro atividades ou menos.
Tabela 4 - Comparação das médias das facetas de QV do instrumento WHOQOL-Old em função dasvariáveis "atividades de lazer", "apoio familiar" e "trabalho remunerado após a aposentadoria" $(\mathrm{n}=169)$

\begin{tabular}{|c|c|c|c|}
\hline \multirow[t]{3}{*}{ Facetas } & \multicolumn{2}{|c|}{ Variável independente } & \multirow[t]{2}{*}{ p-valor } \\
\hline & \multicolumn{2}{|c|}{ Atividades de lazer } & \\
\hline & 0 a 4 & $5 \mathrm{ou}+$ & \\
\hline $\begin{array}{l}\text { Funcionamento dos } \\
\text { sentidos }\end{array}$ & $66,04 \pm 22,01$ & $76,98 \pm 199$ & $0,002 * *$ \\
\hline Autonomia & $63,55 \pm 16,63$ & $71,25 \pm 17,64$ & $0,009 * *$ \\
\hline $\begin{array}{l}\text { Atividades passadas, } \\
\text { presentes e futuras }\end{array}$ & $72,35 \pm 14,88$ & $75,44 \pm 14,54$ & 0,298 \\
\hline Participação Social & $66,68 \pm 18,55$ & $73,85 \pm 15,89$ & $0,000 * *$ \\
\hline Morte e morrer & $68,77 \pm 26,61$ & $69,73 \pm 28,55$ & 0,884 \\
\hline Intimidade & $66,90 \pm 25,65$ & $69,38 \pm 25,66$ & 0,368 \\
\hline \multirow[t]{3}{*}{ Global } & $67,69 \pm 12,17$ & $72,79 \pm 13,93$ & $0,018 *$ \\
\hline & \multicolumn{2}{|c|}{ Ter filhos } & \\
\hline & Sim & Não & \\
\hline $\begin{array}{l}\text { Funcionamento dos } \\
\text { sentidos }\end{array}$ & $69,16 \pm 21,64$ & $73,70 \pm 23,36$ & 0,423 \\
\hline Autonomia & $65,63 \pm 17,18$ & $74,30 \pm 15,92$ & 0,127 \\
\hline $\begin{array}{l}\text { Atividades passadas, } \\
\text { presentes e futuras }\end{array}$ & $73,62 \pm 14,87$ & $70,60 \pm 13,86$ & 0,627 \\
\hline Participação Social & $70,06 \pm 17,63$ & $77,50 \pm 18,88$ & 0,247 \\
\hline Morte e morrer & $69,95 \pm 26,59$ & $60,60 \pm 32,21$ & 0,425 \\
\hline Intimidade & $67,61 \pm 25,67$ & $72,50 \pm 23,74$ & 0,654 \\
\hline \multirow[t]{3}{*}{ Global } & $69,33 \pm 12,80$ & $71,50 \pm 13,64$ & 0,605 \\
\hline & \multicolumn{2}{|c|}{ Morar com filhos } & \\
\hline & Sim & Não & \\
\hline $\begin{array}{l}\text { Funcionamento dos } \\
\text { sentidos }\end{array}$ & $69,16 \pm 20,99$ & $69,30 \pm 22,12$ & 0,805 \\
\hline Autonomia & $62,17 \pm 16,97$ & $67,79 \pm 17,20$ & 0,053 \\
\hline $\begin{array}{l}\text { Atividades passadas, } \\
\text { presentes e futuras }\end{array}$ & $73,75 \pm 15,00$ & $73,33 \pm 14,88$ & 0,752 \\
\hline Participação Social & $70,73 \pm 17,18$ & $69,22 \pm 18,23$ & 0,566 \\
\hline Morte e morrer & $66,72 \pm 28,00$ & $71,69 \pm 25,91$ & 0,355 \\
\hline Intimidade & $68,97 \pm 26,64$ & $66,24 \pm 25,21$ & 0,570 \\
\hline \multirow[t]{3}{*}{ Global } & $68,55 \pm 13,56$ & $69,60 \pm 12,51$ & 0,620 \\
\hline & \multicolumn{2}{|c|}{$\begin{array}{c}\text { Cooperação financeira } \\
\text { familiar }\end{array}$} & \\
\hline & Sim & Não & \\
\hline $\begin{array}{l}\text { Funcionamento dos } \\
\text { sentidos }\end{array}$ & $67,21 \pm 23,59$ & $71,10 \pm 20,17$ & 0,056 \\
\hline Autonomia & $63,21 \pm 17,41$ & $67,92 \pm 17,01$ & 0,056 \\
\hline $\begin{array}{l}\text { Atividades passadas, } \\
\text { presentes e futuras }\end{array}$ & $74,72 \pm 14,36$ & $72,37 \pm 15,09$ & 0,531 \\
\hline Participação Social & $70,15 \pm 18,16$ & $70,43 \pm 17,75$ & 0,801 \\
\hline Morte e morrer & $68,76 \pm 23,92$ & $69,29 \pm 29,30$ & 0,253 \\
\hline Intimidade & $67,51 \pm 26,63$ & $67,81 \pm 25,00$ & 0,799 \\
\hline Global & $68,55 \pm 12,85$ & $69,79 \pm 13,03$ & 0,562 \\
\hline
\end{tabular}


Silva MO, et al. Trabalho, atividades de lazer e apoio familiar. Rev Ter Ocup Univ São Paulo. 2017 maio/ago.;28(2):163-72.

Tabela 4 - Comparação das médias das facetas de QV do instrumento WHOQOL-Old em função dasvariáveis "atividades de lazer", "apoio familiar" e "trabalho remunerado após a aposentadoria" (n=169)

\begin{tabular}{|c|c|c|c|}
\hline \multirow[t]{2}{*}{ Facetas } & \multicolumn{2}{|c|}{ Variável independente } & \multirow[t]{2}{*}{ p-valor } \\
\hline & \multicolumn{2}{|c|}{ Convívio Familiar } & \\
\hline & Ótimo/bom & Regular/ruim & \\
\hline $\begin{array}{l}\text { Funcionamento dos } \\
\text { sentidos }\end{array}$ & $71,75 \pm 20,80$ & $52,95 \pm 20,66$ & $0,001 * *$ \\
\hline Autonomia & $67,18 \pm 17,12$ & $55,33 \pm 15,23$ & $0,009 * *$ \\
\hline $\begin{array}{l}\text { Atividades passadas, } \\
\text { presentes e futuras }\end{array}$ & $74,64 \pm 14,10$ & $63,11 \pm 14,43$ & $0,002 * *$ \\
\hline Participação Social & $71,89 \pm 17,38$ & $57,16 \pm 15,08$ & $0,001 * *$ \\
\hline Morte e morrer & $69,02 \pm 27,23$ & $67,79 \pm 27,35$ & 0,751 \\
\hline Intimidade & $68,85 \pm 16,07$ & $58,84 \pm 10,17$ & $0,032 * *$ \\
\hline \multirow[t]{3}{*}{ Global } & $70,64 \pm 12,73$ & $59,11 \pm 9,72$ & $0,000 *$ \\
\hline & \multicolumn{2}{|c|}{ Situação conjugal } & \\
\hline & $\begin{array}{l}\text { Vive com o(a) } \\
\text { parceiro(a) }\end{array}$ & $\begin{array}{l}\text { Vive sem o(a) } \\
\text { parceiro(a) }\end{array}$ & \\
\hline $\begin{array}{l}\text { Funcionamento dos } \\
\text { sentidos }\end{array}$ & $70,31 \pm 20,41$ & $67,38 \pm 23,80$ & 0,644 \\
\hline Autonomia & $65,33 \pm 18,39$ & $67,36 \pm 15,10$ & 0,717 \\
\hline $\begin{array}{l}\text { Atividades passadas, } \\
\text { presentes e futuras }\end{array}$ & $73,65 \pm 15,41$ & $72,56 \pm 13,75$ & 0,695 \\
\hline Participação Social & $70,15 \pm 18,64$ & $70,22 \pm 16,25$ & 0,888 \\
\hline Morte e morrer & $69,57 \pm 28,89$ & $67,64 \pm 23,61$ & 0,198 \\
\hline Intimidade & $73,49 \pm 21,80$ & $56,16 \pm 28,86$ & $0,000 * *$ \\
\hline \multirow[t]{3}{*}{ Global } & $70,42 \pm 13,56$ & $66,85 \pm 11,30$ & 0,096 \\
\hline & \multicolumn{2}{|c|}{ Ocupação remunerada } & \\
\hline & A-T & A-NT & \\
\hline $\begin{array}{l}\text { Funcionamento dos } \\
\text { sentidos }\end{array}$ & $70,16 \pm 22,33$ & $69,11 \pm 21,72$ & \\
\hline Autonomia & $61,91 \pm 17,09$ & $67,57 \pm 16,60$ & 0,871 \\
\hline $\begin{array}{l}\text { Atividades passadas, } \\
\text { presentes e futuras }\end{array}$ & $74,98 \pm 14,33$ & $72,17 \pm 14,87$ & 0,365 \\
\hline Participação Social & $71,16 \pm 16,47$ & $70,18 \pm 18,58$ & 0,839 \\
\hline Morte e morrer & $76,10 \pm 24,90$ & $66,34 \pm 28,31$ & $0,046^{* *}$ \\
\hline Intimidade & $70,94 \pm 27,01$ & $66,55 \pm 24,85$ & 0,139 \\
\hline Global & $71,69 \pm 12,50$ & $68,63 \pm 12,29$ & 0,171 \\
\hline
\end{tabular}

A-T: aposentados que trabalham; A-NT: aposentados que não trabalham; *Comparação de médias conforme o teste t de Student; **Comparação de tendências conforme o Mann Whitney; $\mathrm{p}<0,05$.

Referente ao apoio familiar notou-se que ter filhos, morar com filhos ou receber ajuda financeira de familiares não gerou interferência significativa sobre os domínios e facetas avaliadas. Em contrapartida, o convívio familiar mostrou ser um fator com efeito significativo sobre os escores médios de QV em quase todos os domínios dos instrumentos, de forma que, quando considerado ótimo ou bom pelos idosos, favoreceu escores médios mais altos, com exceção do domínio "morte e morrer" do WHOQOL-Old.
Relativamente ao tópico "viver com um(a) parceiro(a) ou não" os participantes que se enquadraram na primeira alternativa apresentaram escore médio de QV significativamente maior na faceta "intimidade" do WHOQOL-Old. Informações sobre apoio familiar são observadas na Tabela 5.

Tabela 5 - Comparação das médias dos domínios e média global de QV dos instrumentos WHOQOL-Bref e WHOQOL-Old em função do apoio familiar recebido $(n=169)$

\begin{tabular}{|c|c|c|c|}
\hline \multirow{2}{*}{ Domínio/faceta } & \multicolumn{2}{|c|}{ Características } & \multirow{2}{*}{ p-valor } \\
\hline & Média \pm DP & Média \pm DP & \\
\hline & \multicolumn{2}{|c|}{ Ter filhos } & \\
\hline & Sim & Não & \\
\hline \multicolumn{4}{|l|}{ WHOQOL-Bref } \\
\hline Físico & $63,26 \pm 16,38$ & $69,10 \pm 15,28$ & 0,274 \\
\hline Psicológico & $67,77 \pm 14,26$ & $72,90 \pm 17,52$ & 0,409 \\
\hline Relações sociais & $78,83 \pm 15,00$ & $80,20 \pm 11,87$ & 0,967 \\
\hline Meio ambiente & $62,20 \pm 18,27$ & $65,80 \pm 18,27$ & 0,393 \\
\hline Global & $66,88 \pm 19,51$ & $71,20 \pm 19,68$ & 0,400 \\
\hline \multicolumn{4}{|l|}{ WHOQOL-Old } \\
\hline $\begin{array}{l}\text { Funcionamento dos } \\
\text { sentidos }\end{array}$ & $69,16 \pm 21,64$ & $73,70 \pm 23,36$ & 0,423 \\
\hline Autonomia & $65,63 \pm 17,18$ & $74,30 \pm 15,92$ & 0,127 \\
\hline $\begin{array}{l}\text { Atividades passadas, } \\
\text { presentes e futuras }\end{array}$ & $73,62 \pm 14,87$ & $70,60 \pm 13,86$ & 0,627 \\
\hline Participação Social & $70,06 \pm 17,63$ & $77,50 \pm 18,88$ & 0,247 \\
\hline Morte e morrer & $69,95 \pm 26,59$ & $60,60 \pm 32,21$ & 0,425 \\
\hline Intimidade & $67,61 \pm 25,67$ & $72,50 \pm 23,74$ & 0,654 \\
\hline \multirow[t]{3}{*}{ Global } & $69,33 \pm 12,80$ & $71,50 \pm 13,64$ & 0,605 \\
\hline & \multicolumn{2}{|c|}{ Morar com filhos } & \\
\hline & Sim & Não & \\
\hline \multicolumn{4}{|l|}{ WHOQOL-Bref } \\
\hline Físico & $64,11 \pm 16,61$ & $62,47 \pm 16,32$ & 0,539 \\
\hline Psicológico & $67,28 \pm 13,96$ & $68,00 \pm 14,49$ & 0,632 \\
\hline Relações sociais & $78,16 \pm 16,48$ & $79,06 \pm 14,07$ & 0,933 \\
\hline Meio ambiente & $60,53 \pm 14,66$ & $63,20 \pm 14,88$ & 0,381 \\
\hline Global & $65,83 \pm 18,99$ & $67,62 \pm 19,84$ & 0,573 \\
\hline \multicolumn{4}{|l|}{ Whoqol-Old } \\
\hline $\begin{array}{l}\text { Funcionamento dos } \\
\text { sentidos }\end{array}$ & $69,16 \pm 20,99$ & $69,30 \pm 22,12$ & 0,805 \\
\hline Autonomia & $62,17 \pm 16,97$ & $67,79 \pm 17,20$ & 0,053 \\
\hline $\begin{array}{l}\text { Atividades passadas, } \\
\text { presentes e futuras }\end{array}$ & $73,75 \pm 15,00$ & $73,33 \pm 14,88$ & 0,752 \\
\hline Participação Social & $70,73 \pm 17,18$ & $69,22 \pm 18,23$ & 0,566 \\
\hline Morte e morrer & $66,72 \pm 28,00$ & $71,69 \pm 25,91$ & 0,355 \\
\hline Intimidade & $68,97 \pm 26,64$ & $66,24 \pm 25,21$ & 0,570 \\
\hline \multirow[t]{3}{*}{ Global } & $68,55 \pm 13,56$ & $69,60 \pm 12,51$ & 0,620 \\
\hline & \multicolumn{2}{|c|}{$\begin{array}{c}\text { Cooperação financeira } \\
\text { familiar }\end{array}$} & \\
\hline & Sim & Não & \\
\hline \multicolumn{4}{|l|}{ WHOQOL-Bref } \\
\hline Físico & $61,14 \pm 16,79$ & $65,18 \pm 15,91$ & 0,113 \\
\hline Psicológico & $67,87 \pm 14,59$ & $78,07 \pm 15,20$ & 0,923 \\
\hline Relações sociais & $79,77 \pm 14,41$ & $68,12 \pm 14,42$ & 0,551 \\
\hline
\end{tabular}


Tabela 5 - Comparação das médias dos domínios e média global de QV dos instrumentos WHOQOL-Bref e WHOQOL-Old em função do apoio familiar recebido $(\mathrm{n}=169)$

\begin{tabular}{|c|c|c|c|}
\hline \multirow{2}{*}{ Domínio/faceta } & \multicolumn{2}{|c|}{ Características } & \multirow{2}{*}{ p-valor } \\
\hline & Média \pm DP & Média \pm DP & \\
\hline Meio ambiente & $62,30 \pm 13,25$ & $62,38 \pm 16,21$ & 0,961 \\
\hline Global & $68,14 \pm 19,19$ & $66,44 \pm 19,73$ & 0,434 \\
\hline WHOQOL-Old & & & \\
\hline $\begin{array}{l}\text { Funcionamento dos } \\
\text { sentidos }\end{array}$ & $67,21 \pm 23,59$ & $71,10 \pm 20,17$ & 0,056 \\
\hline Autonomia & $63,21 \pm 17,41$ & $67,92 \pm 17,01$ & 0,056 \\
\hline $\begin{array}{l}\text { Atividades passadas, } \\
\text { presentes e futuras }\end{array}$ & $74,72 \pm 14,36$ & $72,37 \pm 15,09$ & 0,531 \\
\hline Participação Social & $70,15 \pm 18,16$ & $70,43 \pm 17,75$ & 0,801 \\
\hline Morte e morrer & $68,76 \pm 23,92$ & $69,29 \pm 29,30$ & 0,253 \\
\hline Intimidade & $67,51 \pm 26,63$ & $67,81 \pm 25,00$ & 0,799 \\
\hline \multirow[t]{3}{*}{ Global } & $68,55 \pm 12,85$ & $69,79 \pm 13,03$ & 0,562 \\
\hline & \multicolumn{2}{|c|}{ Convívio familiar } & \\
\hline & Ótimo/bom & Regular/ruim & \\
\hline \multicolumn{4}{|l|}{ WHOQOL-Bref } \\
\hline Físico & $64,69 \pm 16,62$ & $54 \pm 17,32$ & $0,007 *$ \\
\hline Psicológico & $69,50 \pm 13,80$ & $56,32 \pm 14,43$ & $0,000 * *$ \\
\hline Relações sociais & $80,81 \pm 12,76$ & $62,84 \pm 20,20$ & $0,000 * *$ \\
\hline Meio ambiente & $64,16 \pm 14,07$ & $48,00 \pm 14,63$ & $0,000 * *$ \\
\hline Global & $68,51 \pm 19,38$ & $56,47 \pm 16,86$ & $0,003 * *$ \\
\hline \multicolumn{4}{|l|}{ WHOQOL Old } \\
\hline $\begin{array}{l}\text { Funcionamento dos } \\
\text { sentidos }\end{array}$ & $71,75 \pm 20,80$ & $52,95 \pm 20,66$ & 0,001 \\
\hline Autonomia & $67,18 \pm 17,12$ & $55,33 \pm 15,23$ & 0,009 \\
\hline $\begin{array}{l}\text { Atividades passadas, } \\
\text { presentes e futuras }\end{array}$ & $74,64 \pm 14,10$ & $63,11 \pm 14,43$ & $0,002 * *$ \\
\hline Participação Social & $71,89 \pm 17,38$ & $57,16 \pm 15,08$ & $0,001 * *$ \\
\hline Morte e morrer & $69,02 \pm 27,23$ & $67,79 \pm 27,35$ & 0,751 \\
\hline Intimidade & $68,85 \pm 16,07$ & $58,84 \pm 10,17$ & $0,032 * *$ \\
\hline \multirow[t]{3}{*}{ Global } & $70,64 \pm 12,73$ & $59,11 \pm 9,72$ & $0,000^{*}$ \\
\hline & \multicolumn{2}{|c|}{ Situação conjugal } & \\
\hline & $\begin{array}{c}\text { Vive com o(a) } \\
\text { parceiro(a) }\end{array}$ & $\begin{array}{c}\text { Vive sem o(a) } \\
\text { parceiro(a) }\end{array}$ & \\
\hline \multicolumn{4}{|l|}{ WHOQOL Bref } \\
\hline Físico & $64,73 \pm 16,62$ & $60,86 \pm 15,68$ & 0,146 \\
\hline Psicológico & $67,77 \pm 13,85$ & $68,29 \pm 15,67$ & 0,670 \\
\hline Relações sociais & $79,28 \pm 15,19$ & $77,78 \pm 14,39$ & 0,412 \\
\hline Meio ambiente & $62,83 \pm 15,18$ & $61,53 \pm 14,84$ & 0,855 \\
\hline Global & $66,71 \pm 18,92$ & $67,64 \pm 20,53$ & 0,502 \\
\hline \multicolumn{4}{|l|}{ WHOQOL Old } \\
\hline $\begin{array}{l}\text { Funcionamento dos } \\
\text { sentidos }\end{array}$ & $70,31 \pm 20,41$ & $67,38 \pm 23,80$ & 0,644 \\
\hline Autonomia & $65,33 \pm 18,39$ & $67,36 \pm 15,10$ & 0,717 \\
\hline $\begin{array}{l}\text { Atividades passadas, } \\
\text { presentes e futuras }\end{array}$ & $73,65 \pm 15,41$ & $72,56 \pm 13,75$ & 0,695 \\
\hline Participação Social & $70,15 \pm 18,64$ & $70,22 \pm 16,25$ & 0,888 \\
\hline Morte e morrer & $69,57 \pm 28,89$ & $67,64 \pm 23,61$ & 0,198 \\
\hline Intimidade & $73,49 \pm 21,80$ & $56,16 \pm 28,86$ & $0,000 * *$ \\
\hline Global & $70,42 \pm 13,56$ & $66,85 \pm 11,30$ & 0,096 \\
\hline
\end{tabular}

*Comparação de médias conforme o teste $t$ de Student; DP: Desvio padrão; **Comparação de tendências centrais conforme o Mann Whitney; $\mathrm{p}<0,05$.

\section{DISCUSSÃO}

A predominância de mulheres idosas no estudo é corroborada pela literatura, retratando a feminização da velhice. A maior expectativa de vida das mulheres em relação aos homens se dá pela menor exposição a fatores de risco de mortalidade por causas externas, menor exposição a drogas lícitas como álcool e tabaco, e diferença de posturas quanto ao cuidado da própria saúde ${ }^{21,22,23}$.

Verificou-se que a faixa etária predominante equivalente aos idosos de 60 aos 69 anos de idade se aproximou da média encontrada em um estudo realizado em Uberaba, centro urbano de referência em saúde para a região de Sacramento, que indicou faixa mais prevalente de 60 a 70 anos de idade entre idosos frequentadores de um centro de convivência ${ }^{23}$.

Ao serem considerados os anos de estudo observou-se que a maior parte da amostra possui nível de escolaridade relativamente baixo, sem ultrapassar quatro anos, o que corrobora com o perfil de idosos do Brasil, no qual a proporção da população na terceira idade com ensino médio e superior é inferior à que possui apenas o ensino fundamental, muitas vezes incompleto ${ }^{22,24}$. O número de idosos sem estudo também é preocupante, uma vez que melhores níveis de escolaridade contribuem diretamente para o processo de inclusão social ${ }^{22}$. Notou-se que a renda familiar dos idosos oscila predominantemente entre um e três SM, fator equivalente ao encontrado em um estudo que avaliou a QV de idosos de uma estratégia de saúde da família (ESF) numa cidade do Paraná ${ }^{24}$.

Com o envelhecimento, os idosos passam por um progressivo processo de perdas de papéis nos diferentes ambientes em que habitam com repercussão sobre a QV. Para amenizar e facilitar a passagem por esta etapa, a substituição de papéis e atividades por outras consideradas significativas aumenta os sentimentos de utilidade social e bem-estar ${ }^{3}$.

Considerando a atual realidade sociodemográfica do idoso no Brasil, percebe-se que frequentemente o idoso é responsável pela manutenção financeira ou complementação da renda da família, usualmente composta por diferentes gerações. Devido a isto, ele permanece por mais tempo no mercado de trabalho, predominantemente em serviços informais que demandam maior desgaste físico e emocional ${ }^{22,23}$.

Avaliando a perspectiva do trabalho remunerado após a aposentadoria como um fator de proteção, observouse que uma parcela significativa de idosos $(31,0 \%)$ exerce alguma atividade remunerada, mesmo após o alcance da aposentadoria. Tais idosos apresentaram média 
significativamente maior no domínio físico do WHOQOL. A atividade laboral envolve mecanismos de competição, que podem ser benéficos, pois implicam desafios e auxiliam na manutenção da capacidade funciona ${ }^{10,23}$.

Vale ressaltar que o trabalho é considerado um fator capaz de minimizar o processo de exclusão e marginalização ao qual a população idosa está exposta, por garantir a manutenção das relações sociais que oferecem suporte, cooperação e oportunidades de interatividade e sentimento de utilidade social, gerando sensação de bem-estar ${ }^{9}$. Estas considerações podem ser válidas também para compreender os melhores escores do domínio "morte e morrer" dos idosos que, após a aposentadoria ainda trabalham, já que esse bem-estar pode interferir na maneira de lidar com o processo de envelhecimento e nas estratégias de enfrentamento do medo da morte e de sentimentos negativos a ela associados.

Além desses efeitos positivos, constatou-se que o número de atividades de lazer geralmente influencia a QV, potencializando os aspectos relacionados ao bem-estar físico, psicológico e sensorial e aumentando a capacidade do idoso de responder por suas decisões com maior autonomia. Este indivíduo é capaz de se apropriar melhor dos espaços e das relações sociais que o cercam, fato que fortalece sua rede de suporte.

A literatura aborda os benefícios físico-funcionais e psicoemocionais que o lazer pode proporcionar, não explorando, entretanto, como o mesmo pode interferir nos aspectos sensoriais dos idosos ${ }^{21,23}$. Neste estudo observouse que o engajamento em atividades de lazer propicia aumento do senso de autoeficácia referente ao julgamento sobre habilidades de desempenhar uma atividade e o comportamento com êxito na busca de um objetivo, que, por sua vez, favorece a adoção de comportamentos promotores de saúde, especialmente quando estes geram no sujeito sentimentos de prazer e satisfação ${ }^{24}$, compondo um ciclo de retroalimentação. O lazer é um fator promotor de saúde em virtude do bem-estar gerado em sua execução, melhorando, consequentemente, a percepção da QV da população de idosos ${ }^{3}$.

As atividades são classificadas em humanas e naturais, sendo que as primeiras exigem um planejamento prévio e objetivo específico, diferente das segundas, que possuem origem mais instintiva, direcionadas à sobrevivência do indivíduo. As atividades humanas possibilitam a expressão da singularidade individual no momento em que o homem interage com seu fazer, o que possibilita trocas sociais e combate do isolamento. Dentre tais atividades, encontram-se as aquelas relativas ao lazer, realizadas não como alternativas para preencher o tempo ocioso, mas voltadas para um fazer instituído de desejos ${ }^{25}$.

Vale ressaltar que as atividades de lazer sofrem influência cultural, pois irão variar de região para região de acordo com os costumes e preferências da população local. Essas atividades também variam conforme as faixas etárias e interesses, já que com o avançar da idade, a ampliação de dificuldades e mudança de oportunidades, o interesse e a motivação podem ser modificados. As atividades de lazer podem ainda ser classificadas em interesses: manuais, intelectuais, físicos, artísticos, turísticos e sociais ${ }^{27}$. Isto pôde ser observado quando, nas respostas dos idosos sobre atividades de lazer presentes em suas vidas não incluídas no questionário fechado, foram citadas atividades como envolvimento na produção de artesanatos e composições artísticas, viagens, ida para ranchos e sítios, cultivo de plantas, visitas a festas populares promovidas pela comunidade, dentre outras, fato que reflete as influências culturais da região transmitidas por meio das gerações. Assim, é importante para os profissionais que trabalham na atenção ao idoso, investigar as preferências do mesmo a fim de articular e divulgar ações e encaminhá-los para programas que ofertem contato com essas atividades.

Um bom convívio familiar pode apresentar reflexos positivos na QV dos idosos, fato que vai além do suporte físico e econômico. Isto é pontuado uma vez que os sujeitos que relataram usufruir dessa relação de forma positiva apresentaram escores significativamente maiores nos domínios físico, psicológico, relações sociais, meio ambiente do WHOQOL-Bref e nas facetas funcionamento dos sentidos, autonomia, atividades passadas presentes e futuras, participação social e intimidade do WHOQOLOld, além de melhores dos escores globais nos dois instrumentos utilizados.

O apoio da família ao idoso é fundamental, pois possibilita o sentimento de competência social, maior facilidade no enfrentamento e resolução de problemas, percepção de controle, senso de estabilidade, autoconceito, afeto e bem-estar'.

Enfatiza-se que já existem programas e serviços implementados a partir das políticas públicas com o objetivo de fortalecer os vínculos familiares e assegurar os direitos dos idosos e de suas famílias, contribuindo para a autonomia, participação social e promoção do bem-estar de ambos. Dentre estes, destacam-se programas implementados pelo Serviço Único de Assistência Social (SUAS), como o Serviço de Proteção e Atendimento Integral à Família (PAIF), o Serviço de Convivência e Fortalecimentos de Vínculos, Serviço de Proteção Básico no Domicílio, e o Serviço de Proteção Social Especial para 
Pessoas com Deficiência ${ }^{4}$. A oferta e divulgação desses serviços são imprescindíveis para a ativação da rede e articulação dos profissionais de saúde e assistência social envolvidos no processo de cuidado do idoso e sua família, visando atendimento integral, englobando inclusive a esfera socioafetiva.

\section{CONCLUSÃO}

Os resultados deste estudo permitem concluir que maior repertório de atividades de lazer significativas, bom convívio familiar e manutenção de trabalho remunerado após a aposentadoria comportam-se como fatores protetivos para a $\mathrm{QV}$ dos idosos entrevistados em diferentes esferas e aspectos que compõem a avaliação deste conceito complexo e subjetivo.

É necessário o empenho dos profissionais e gestores em oferecer e sistematizar serviços e oportunidades que otimizem o engajamento em atividades de lazer e trabalho, resgatando o valor social e a construção de projetos de vida na velhice. Ademais, as dinâmicas dos serviços devem ser orientadas à atenção integral visando favorecer o fortalecimento dos vínculos familiares e fazer com que a família se torne um espaço reprodutor de papéis ocupacionais.

Foram observados como pontos positivos do estudo: a existência de amostra representativa do município, ou seja, a amostra contou com um número significativo de sujeitos considerando a população geral do município e esses sujeitos mostraram-se capazes de responder com autonomia às questões avaliadas. Acrescenta-se como outro ponto positivo o emprego de instrumentos específicos e cientificamente reconhecidos para avaliar a QV. Ainda os avaliadores receberam capacitação prévia para aplicação dos testes e questionários de forma padronizada para diminuir chances de viés.

Contrariamente, o corte transversal do estudo não permitiu determinar a verdadeira relação de causa e efeito dos fatores protetivos das variáveis sobre a QV dos idosos. Logo, sugere-se serem desenvolvidos estudos longitudinais que verifiquem o efeito do engajamento em atividades de lazer, do bom convívio familiar e do trabalho após a aposentadoria na QV de grupos de idosos a médios e longos prazos.

Contribuição dos autores: Maysa de Oliveira Silva - Participou na idealização do estudo, coleta dos dados e preparação do artigo para divulgação dos resultados; Álvaro da Silva Santos - Participou da construção do projeto, submissão a órgão de fomento e organização da coleta dos dados; Lucila Costa Zini Angelotti - Participou do delineamento metodológico do estudo; Valéria Sousa de Andrade Participou das análises metodológicas e na preparação do estudo; Grasielle Silveira Tavares - Participou na idealização, orientação e preparação do artigo para divulgação dos resultados.

\section{REFERÊNCIAS}

1. Pereira RJ, Cotta RMM, Franceschini SCC, Ribeiro RCL, Sampaio RF, Priore ES, et. al. Influência de fatores sócios sanitários na qualidade de vida dos idosos de um município do Sudeste do Brasil. Cien Saude Coletiva. 2011;16(6):290717. doi: 10.1590/S0080-623420130000500016.

2. Rodrigues MAP, Facchini LA, Piccini RX, Tomasi E, Thumé E, Silveira DS, et. al. Uso de serviços básicos de saúde por idosos portadores de condições crônicas, Brasil. Rev Saude Publica. 2009;43(4):604-12. doi: 10.1590/S003489102009005000037.

3. Souza LM, Lautert L, Hilleshein EF. Qualidade de vida e trabalho voluntário em idosos. Rev Esc Enferm USP. 2011;45(3):665-71. doi: 10.1590/S0080-62342011000300017.

4. Bulla LC, Tsuruzono ERS. Envelhecimento, família e políticas sociais. Rev Pol Publicas (São Luis). 2010;14(1):103-12.
Disponível em: http://www.periodicoseletronicos.ufma.br/ index.php/rppublica/article/view/358/777.

5. Ribeiro JP, Rocha SA, Popim RC. Compreendendo o significado de qualidade e vida segundo idoso portadores de diabetes mellitus tipo II. Esc Anna Nery. 2010;14(4):765-71. doi: 10.1590/S1414-81452010000400016.

6. Murakami L, Scattolin F. Avaliação da independência funcional e da qualidade de vida de idosos institucionalizados. Rev Med Hered. 2010;21(1):18-26. doi: 10.20453/rmh.v21i1.1141.

7. Organização Mundial de Saúde (OMS). Envelhecimento ativo: uma política de saúde. Brasília: Organização PanAmericana da Saúde; 2005.

8. Freitas CASL, Silva MJ, Vieira NFC, Ximenes LB, Brito MCC, Gubert FA. Evidências de ações de enfermagem em 
promoção da saúde para um envelhecimento ativo: revisão integrativa. Estud Interdiscip Envelhec. 2010;15(2):26577. Disponível em: http://www.seer.ufrgs.br/index.php/ RevEnvelhecer/article/view/12836/11549.

9. Areosa SVC, Benitez LB, Wichmann FMA. Relações familiares e o convívio social entre idosos. Texto Contexto (Porto Alegre). 2012;11(1):184-92. Disponível em: https:// core.ac.uk/download/pdf/27226830.pdf.

10. D’Orsi E, Xavier AJ, Ramos LR. Trabalho, suporte social e lazer protegem idosos da perda funcional: estudo Epidoso. Rev Saude Publica. 2011;45(4):685-92. doi: 10.1590/S003489102011000400007.

11. Bulla LC, Kaefer CO. Trabalho e aposentadoria: as repercussões sociais na vida do idoso aposentado. Textos Contextos (Porto Alegre). 2003;2(2):1-8. Disponível em: http://revistaseletronicas.pucrs.br/ojs/index.php/fass/article/ view/957/737.

12. Martins, R. Os idosos e as actividades de lazer. Rev Millenium. 2010;38:243-51. Disponível em: http://www. ipv.pt/millenium/Millenium38/16.pdf.

13. Instituto Brasileiro de Geografia e Estatística. Resultados preliminares do censo demográfico 2010 [citado 06 abr. 2009]. Disponível em: http://www.ibge.gov.br/ home/estatistica/populacao/censo2010/resultados preliminares_amostra/notas_resultados_preliminares_ amostra.pdf

14. Bertolucci PHF, Brucki SMD, Campacci SR, Juliano Y. O Mini-Exame do Estado Mental em uma população geral: impacto da escolaridade. Arq Neuropsiquiatr. 1994;52(1):330-5. doi: 10.1590/S0004282X1994000100001.

15. Harper A, Power M. Development of the World Health Organization WHOQOL-BREF quality of life assessment. Psychol Med. 1998;28(3):551-8. doi: 10.1017/ S0033291798006667.

16. Power M, Schmidt S, WHOQOL-OLD Group. Development of the WHOQOL-old module. Qual Life Res. 2005;14(10):2197-214. doi: 10.1007/s11136-005-7380-9
17. Fleck MPA, Louzada S, Xavier M, Chachamovich E, Vieira $\mathrm{G}$, Santos L, et al. Aplicação da versão em português do instrumento abreviado de avaliação da qualidade de vida "WHOQOL-bref". Rev Saude Publica. 2000;34(2):178-83. doi: 10.1590/S0034-89102000000200012.

18. Fleck MP, Chachamovich E, Trentini C. Development and validation of the Portuguese version of the WHOQOLOLD module. Rev Saude Publica. 2006;40(5):785-91. doi: 10.1590/S0034-89102006000600007.

19. Oliveira ERA, Gomes MJ, Paiva KM. Institucionalização e qualidade de vida de idosos da região metropolitana de Vitória - ES. Esc Anna Nery. 2011;15(3):518-23. doi: 10.1590/S1414-81452011000300011.

20. Manso MEG, Lopes RGC. Avaliação da qualidade de vida de um grupo de idosos portadores de doenças crônicas não transmissíveis vinculados a um programa de promoção da saúde. Rev Bras Med. 2010;67(esp.5):12-19. doi: 10.13140/2.1.2184.7843.

21. Caetano ACM, Tavares DMS. Unidade de atenção ao idoso: atividades, mudanças no cotidiano e sugestões. Rev Eletr Enf. 2008;10(3):622-31. doi: 10.5216/ree.v10.46592.

22. Faller JW, Melo WA, Versa GLGS, Marcon SS. Qualidade de vida de idosos cadastrados na estratégia saúde da família de Foz do Iguaçu-PR. Esc Anna Nery. 2010;14(4):803-10. doi: 10.1590/S1414-81452010000400021.

23. Tiveron RM. A terapia ocupacional no campo da gerontologia: uma contribuição para revisão de projetos de vida [dissertação]. São Paulo: Pontifícia Universidade Católica de São Paulo; 2008. Disponível em: https://tede2. pucsp.br/handle/handle/12546.

24. Silva MCS, Lautert L. O senso de autoeficácia na manutenção de comportamentos promotores de saúde de idosos. Rev Esc Enferm USP. 2010;44(1):61-7. doi: 10.1590/S0080-62342010000100009.

25. Reis N, Soares T. Lazer e envelhecimento saudável: um recorte sobre a relevância dos conteúdos culturais. Lect Educ Fis Deportes. 2006;99(1):18. Disponível em: http:// www.efdeportes.com/efd99/lazer.htm. 